\title{
On the relation between ID-Logic and Answer Set Programming *
}

\author{
Maarten Mariën ${ }^{1}$, David Gilis ${ }^{1}$, and Marc Denecker ${ }^{1}$ \\ Department of Computer Science, Katholieke Universiteit Leuven, Belgium \\ \{Maarten.Marien, David.Gilis, Marc.Denecker\}@cs.kuleuven.ac.be
}

\begin{abstract}
This paper is an analysis of two knowledge representation extensions of logic programming, namely Answer Set Programming and ID-Logic. Our aim is to compare both logics on the level of declarative reading, practical methodology and formal semantics. At the level of methodology, we put forward the thesis that in many (but not all) existing applications of ASP, an ASP program is used to encode definitions and assertions, similar as in ID-Logic. We illustrate this thesis with an example and present a formal result that supports it, namely an equivalence preserving translation from a class of ID-Logic theories into ASP. This translation can be exploited also to use the current efficient ASP solvers to reason on ID-Logic theories and it has been used to implement a model generator for ID-Logic.
\end{abstract}

\section{Introduction}

This paper is a comparison of Answer Set Programming [9,12], more precisely, of General Logic Programming [8] or Stable Logic Programming [11], and IDLogic $[2,6]$. Both logics can be considered as extensions of logic programming for knowledge representation. The basic formal result of this paper is an equivalence preserving translation from an important class of ID-Logic theories to ASP. This result leads to improved understanding of these logics in different ways. Not only does it give insight in the formal relationships between the logics, but it also leads to improved understanding of the methodology of ID-Logic and ASP and allows to compare them. Moreover, this result can be exploited to use the current generation of efficient ASP solvers to reason on or perform problem solving using ID-Logic theories. In fact, we discuss an existing model generator for ID-Logic which we built using this translation and the Smodels system.

ID-Logic is an extension of classical first order logic that allows for a uniform representation of various forms of definitions, including non-inductive definitions, monotone inductive definitions (e.g. the transitive closure of a graph) and nonmonotone forms of inductive definitions such as iterated induction and induction over well-founded posets (e.g. the standard definition of truth of a formula in a structure). An ID-Logic theory consists of a set of FOL sentences, called

\footnotetext{
* Works supported by FWO-Vlaanderen, European Framework 5 Project WASP, and
} by GOA/2003/08. 
assertions, and definitions. A definition is represented as a set of definitional rules of the form $\forall \bar{x}(A \leftarrow \alpha)$ where $A$ is an atom, $\leftarrow$ the so-called definitional implication (to be distinguished from material implication, $\subset$ ) and $\alpha$ a FOL formula (thus negation in ID-Logic is classical negation). A definition defines a set of defined predicates, namely those occurring in the head of rules, in terms of other open predicates, which appear only in the body of rules. Note that an ID-Logic theory never contains a definitional rule; it only may contain sets of such rules. The formal semantics is an integration of classical logic semantics and the well-founded semantics for definitions. Formally, Abductive Logic Programming under well-founded semantics can be seen as the subformalism of ID-Logic consisting of theories with only one definition and imposing the Domain Closure Axiom and the Unique Name Axioms. In [4], a extension called NMID-logic was proposed allowing for arbitrary boolean combinations of definitions and FOL formulas. The same paper explores the use of this logic for knowledge representation in the context of situation calculus.

The second formalism that we consider here is General Logic Programming [8] or Stable Logic Programming as it was called in [11,12]. A program in this formalism consists of general program rules of the form $A \leftarrow B o d y$ where $A$ is an atom and Body is a conjunction of literals $B$ or not $B$ where $B$ is an atom. The semantics is the stable model semantics. The formalism is a subformalism of Answer Set Programming (ASP), without the strong negation and the disjunction in the head. Despite these limitations, most applications of ASP can be represented in it or in its extension with weight constraints [12]. The formalism is generally seen as a sublogic of default logic and negation as failure as a default negation operator "it is possible to assume that $A$ is false".

Both logics show considerable differences on the conceptual, syntactical and semantical level. Yet, if we compare examples and methodologies, striking similarities show up. To illustrate this, we take a representation of the wellknown notion of hamiltonian cycles of a graph. (See Figure 1, where we implicitly assume that the unique names axioms and the domain closure axioms hold.)

There is an apparent similarity on the level of clauses and structure of the theory. In both theories, four different parts can be distinguished:

- data, representing the graph by a set of atomic clauses in ASP or by two definitions of Vertex/1 and Edge/2 in ID-Logic.

- ASP rules to open up predicates, here only the predicate in $/ 2$. In ASP, this can be done also using a disjunction (as in dlv) or using a weight constraint (as in Smodels). Often, such rules specify also a domain for the opened predicate. The domain of $i n / 2$ is the predicate edge/2 which means that $\mathrm{in} / 2$ is a subset of edge/1. As ID-Logic is an extension of classical logic, predicates are open per default; the domain declaration is formalised by an implication.

- definitions, here only of the concept of reachable vertices (through hamiltonian edges).

- assertions, (called constraints in ASP) representing the basic properties of hamiltonian cycles. 


\begin{tabular}{|c|c|}
\hline ID-Logic & ASP (taken from [11]) \\
\hline $\begin{array}{l}\left\{\begin{array}{l}\text { Vertex }(U) \leftarrow\} \\
\ldots\end{array}\right\} \\
\left\{\begin{array}{l}\operatorname{Edge}(U, V) \leftarrow\} \\
\ldots\end{array}\right. \\
\{\text { InitialVtx }(U) \leftarrow\}\end{array}$ & $\begin{array}{l}\text { vertex }(u) \leftarrow \\
\ldots \\
\text { edge }(u, v) \leftarrow \\
\ldots \\
\text { initialvtx }(u) \leftarrow\end{array}$ \\
\hline & $\begin{array}{l}\operatorname{in}(V 1, V 2) \leftarrow \\
\quad \text { edge }(V 1, V 2), \text { not out }(V 1, V 2) \\
\text { out }(V 1, V 2) \leftarrow \\
\quad \operatorname{edge}(V 1, V 2), \text { not } \operatorname{in}(V 1, V 2)\end{array}$ \\
\hline$\left\{\begin{array}{c}\forall x, y(\text { Reached }(x) \leftarrow \\
\operatorname{In}(y, x) \wedge \text { InitialVtx }(y)) \\
\forall x, y(\text { Reached }(x) \leftarrow \\
\operatorname{In}(y, x) \wedge \operatorname{Reached}(y))\end{array}\right\}$ & $\begin{array}{l}\text { reached }(V 2) \leftarrow \\
\qquad \begin{array}{l}\text { in }(V 1, V 2), \text { reached }(V 1) \\
\text { reached }(V 2) \leftarrow \\
\text { in }(V 1, V 2), \text { initialvt } x(V 1)\end{array} \\
\end{array}$ \\
\hline $\begin{array}{l}\forall x, y(\operatorname{In}(x, y) \supset \operatorname{Edge}(x, y)) \\
\forall x, y, z((\operatorname{In}(x, y) \wedge \operatorname{In}(x, z)) \supset(y=z)) \\
\forall x, y, z((\operatorname{In}(y, x) \wedge \operatorname{In}(z, x)) \supset(y=z)) \\
\forall x(\operatorname{Vertex}(x) \supset \operatorname{Reached}(x))\end{array}$ & $\begin{array}{c}f \leftarrow i n(V 2, V 1), i n(V 3, V 1) \\
\operatorname{not} V 2=V 3, \operatorname{not} f \\
f \leftarrow i n(V 1, V 2), i n(V 1, V 3) \\
\operatorname{not} V 2=V 3, \operatorname{not} f \\
f \leftarrow \operatorname{not} \operatorname{reached}(X), \operatorname{not} f\end{array}$ \\
\hline
\end{tabular}

Fig. 1. Hamiltonian circuit

Note that negation as failure in the ASP program corresponds to classical negation in the ID-Logic theory. This shows that in this type of ASP programs, negation as failure is to be interpreted as classical negation.

The role of definitional and assertional knowledge for knowledge representation has long been recognised in AI [18,1] and was the motivation for Description logics. The distinction between both sorts of knowledge is a fundamental one which sheds light on certain aspects of ASP methodology that are otherwise hard to explain. For example, we need to express that in a hamiltonian cycle, all vertices are reachable. In ID-Logic this property is expressed by the FOL axiom $\forall x(\operatorname{Vertex}(x) \supset$ Reached $(x))$. In the ASP program, this is expressed by the constraint $f \leftarrow$ not reached $(X)$, not $f$. Consider an alternative representation by the rule reached $(X) \leftarrow \operatorname{vertex}(X)$. If we use this representation, we actually get models in which no edge belongs to the hamiltonian cycle. How can 
we explain this? The reason is that ASP interprets reached $(X) \leftarrow \operatorname{vertex}(X)$ as an additional definitional rule, while in fact, it represents assertional knowledge. The only correct way to represent such knowledge in ASP, is by constraints such as $f \leftarrow \operatorname{not}$ reached $(X)$, not $f$.

In our experience, the pattern of four parts consisting of data, declarations of open predicates, definitions and assertions, can be found in most applications of Stable Logic Programming. Other examples can be found in $[13,14]$, in which different LP-approaches to KR are compared. The thesis that we want to launch in this paper, is that SLP can be interpreted as a language for representing definitions and assertions and moreover that this explains most applications and the methodology that is commonly used in ASP.

Of course, this thesis cannot be formalised or formally proven. However, the rest of this paper is concerned with a formal translation from ID-Logic with Unique Names Axioms and the Domain Closure Axiom to general logic programs which provides strong support for the thesis. The translation sheds light on how assertions and definitions are implicitly encoded in ASP. The main problem for proving the correctness of this translation is the use of well-founded semantics in ID-Logic versus stable semantics in ASP: basically we will show that the nondeterminism of multiple stable models of many ASP programs derives from the combination of open predicates with deterministic definitions on top of them.

As a final remark, we do not claim that all ASP programs can be understood as encodings of ID-Logic theories. Certain ASP programs should be understood as autoepistemic theories or default theories, and not as ID-Logic theories. An example taken from [7] is the following rule:

$$
\operatorname{check\_ status}(X) \leftarrow \operatorname{person}(X), \operatorname{not} \operatorname{orphan}(X) \text {, not } \neg \text { orphan }(X)
$$

The intended declarative reading of this rule is that the status of a person should be checked if it is unknown whether it is an orphan or not. In this rule, the negation as failure has indeed a non-objective modality; such a modality is not available in ID-Logic. What this also shows is that in different applications and subsets of ASP, negation as failure and the rule operator have different meanings. This ambiguity is investigated in [4].

\section{Preliminaries}

\subsection{Logic programs}

We first introduce some terminology and basic concepts. A vocabulary $\tau$ is a set of constant, function, predicate and variable symbols. The Herbrand universe of $\tau$, consisting of all terms of $\tau$ is denoted $\mathcal{H U}(\tau)$. A Herbrand interpretation of $\tau$ is a set of ground atoms of $\tau$, containing all atoms that are true. A 3valued Herbrand interpretation will be defined here as a pair $(I, J)$ of Herbrand interpretations such that $I \subseteq J$. Intuitively, an atom $A$ is true in $(I, J)$ if it is true in $I$, it is false in $(I, J)$ if it is false in $J$ and otherwise, it is undefined in 
$(I, J)$. A pair $(I, J)$ is viewed here as a tuple of underestimate $I$ and overestimate $J$.

A general logic program (in $\tau$ ) is a set of clauses of the form $A \leftarrow A_{1} \wedge . \wedge A_{i} \wedge$ not $A_{i+1} \wedge \ldots \wedge$ not $A_{n}$, with $A, A_{i}$ all atoms (in $\tau$ ). We allow infinite programs and infinitary rules (i.e. $i$ and $n$ can be infinite). A definite logic program $P$ is a general logic program without negative literals. A definite logic program has a least Herbrand model denoted $\mathcal{L H} \mathcal{M}(P)$.

The grounding of a general logic program $P$ is defined as usual, as the set of all rules that can be obtained by instantiating variables in rules of $P$ by ground terms. Also usual, the grounding is seen as a propositional logic program.

We recall the stable semantics [8] and well-founded semantics [17] of general logic programs. As usual, we define these semantics for propositional programs only. Semantics of predicate programs are defined through their grounding.

Given a general logic program $P$ in $\tau$ and a Herbrand interpretation $I$. The reduct $P_{I}$ is the program obtained from $P$ by deleting

- each rule that has a negative literal not $q_{i}, q_{i} \in I$ in the body

- all negative literals in the body of the remaining rules.

Since $P_{I}$ is a definite logic program, $\mathcal{L} \mathcal{H} \mathcal{M}\left(P_{I}\right)$ exists.

We define the Gelfond-Lifschitz operator $G L_{P}$ associated to program $P$ as the operator of Herbrand interpretations which maps an interpretation $I$ to $\mathcal{L} \mathcal{H} \mathcal{M}\left(P_{I}\right)$.

A stable model of $P$ is defined as a fixpoint of $G L_{P}$, i.e. as a Herbrand interpretation $I$ such that $I=\mathcal{L} \mathcal{H} \mathcal{M}\left(P_{I}\right)$.

To define the notion of well-founded model of $P$, we follow the approach of [3]. The operator $G L_{P}$ is antimonotone, i.e. if $I \subseteq J$ then $G L_{P}(I) \supseteq G L_{P}(J)$. As a consequence, $G L_{P}^{2}$ is a monotone lattice operator and has a least fixpoint lf $p\left(G L_{P}^{2}\right)$ and a largest fixpoint $g f p\left(G L_{P}^{2}\right)$. The pair $\left(l f p\left(G L_{P}^{2}\right), g f p\left(G L_{P}^{2}\right)\right)$ is the well-founded model of $P$. It holds that for each stable model $I, \operatorname{lf} p\left(G L_{P}^{2}\right) \subseteq$ $I \subseteq g f p\left(G L_{P}^{2}\right)$

\subsection{ID-Logic}

As mentioned in the introduction, an ID-Logic theory $T$ in $\tau$ is a set of FOL sentences and definitions. Each definition $D$ is a set of rules of the form $\forall \bar{x}(A \leftarrow$ $\alpha$ ) where $A$ is an atom and $\alpha$ a FOL formula. Each definition $D$ has a set $\operatorname{Def}(D)$ of defined predicates, i.e., those appearing in the head of a rule. (The defined predicates may explicitly be mentioned in front of the rule set, as in $P / n, Q / m::=\{\ldots\}$, which simultaneously defines the predicate $P$ with arity $n$, and the predicate $Q$ with arity $m$. The empty definition of a predicate $P / n$ can be represented by $P / n::=\{\}$.) The set $\tau \backslash \operatorname{Def}(D)$ is called the set of open symbols of $D$ and is denoted Open $(D)$.

The semantics of ID-Logic is based on an extension of the well-founded semantics to arbitrary (non-Herbrand) interpretations. This extension associates with each definition $D$ and an arbitrary interpretation $I_{o}$ of $O p e n(D)$ a unique 
(possibly 3-valued) well-founded model $I_{o}^{D}$, called the well-founded model of $D$ extending $I_{o}$. A $\tau$-interpretation $I$ is a model of $\boldsymbol{D}$ if $I$ is two-valued and $I=\left(\left.I\right|_{\text {Open }(D)}\right)^{D}$. Here, $\left.I\right|_{\text {Open }(D)}$ denotes the restriction of $I$ to the open symbols of $D$. Formally, an interpretation $I$ is a model of an ID-Logic theory iff it is a model of each of its FOL sentences and a model of each of its definitions $D$. For details we refer to $[2,6]$.

Example 1. Consider the ID-Logic definition $\{P \leftarrow Q\}$. There are two interpretations of the open predicate $Q$ of this definition: one where $Q=\boldsymbol{t}$, one where $Q=\boldsymbol{f}$. The corresponding well-founded models of the definition are resp. $\{P, Q\}$ and $\emptyset$. By a symmetric argument, these interpretations are also the two models of the definition $\{Q \leftarrow P\}$. Consequently, the theory $T_{1}=[\{P \leftarrow Q\},\{Q \leftarrow P\}]$ has models $\{P, Q\}$ and $\emptyset$.

On the other hand, the theory $T_{2}=[\{P \leftarrow Q, Q \leftarrow P\}]$ has only one model: $\emptyset$. The definition has no open predicates and its well-founded model is $\emptyset$.

From a knowledge representation perspective, the use of Herbrand interpretations boils down to the use of the Domain Closure Axiom and the Unique Name Axioms. Those are not imposed by FOL nor by ID-Logic but they can be expressed in ID-Logic [6]. However, in the context of this paper we will only consider ID-Logic theories which (implicitly) contain these axioms. So, all models are Herbrand models (modulo isomorphism).

A crucial notion is that of totality: a definition $D$ is total in an interpretation $I_{o}$ of $\operatorname{Open}(D)$ iff the well-founded model $I_{o}^{D}$ of $D$ extending $I_{o}$ is 2-valued. A definition $D$ is total in a theory $T_{o}$ in $\operatorname{Open}(D)$ if $D$ is total in each model of $T_{o}$. A definition $D$ is total if it is total in the empty theory, that is if $D$ is total in each interpretation $I_{o}$ of $\operatorname{Open}(D)$. Intuitively, a definition $D$ is total in $I_{o}$ if the definition allows to determine the truth values of all the defined atoms in the context of $I_{o}$.

\section{The transformation}

We first present a formal transformation for a restricted subclass of ID-Logic theories which comprises the example of Section 1. In the next subsection, we extend the transformation to more general cases.

We will use the following notion of equivalence. Let $\tau_{1}, \tau_{2}$ be vocabularies extending $\tau$, and $T_{1}$ and $T_{2}$ theories in respectively $\tau_{1}, \tau_{2}$; then $T_{1}$ and $T_{2}$ are equivalent in $\tau$ (denoted $T_{1} \equiv_{\tau} T_{2}$ ) if for each $\tau_{1}$-model $M_{1}$ of $T_{1}$, there exists a $\tau_{2}$-model $M_{2}$ of $T_{2}$ such that $\left.M_{1}\right|_{\tau}=\left.M_{2}\right|_{\tau}$ and vice versa. The theories $T_{1}$ and $T_{2}$ do not necessarily belong to the same logic, e.g., $T_{1}$ might be an ID-Logic theory and $T_{2}$ a stable logic program.

\subsection{A first transformation}

The class of ID-Logic theories $T$ considered here in this section have a similar structure as the example of Section 1. They consist of the following components: 
- definitions $D_{P / m}$ to represent data, defining certain predicates by exhaustive enumeration. Such definitions consist of ground atomic rules;

- one domain declaration $\forall \bar{x}\left(P(\bar{x}) \supset C_{P}(\bar{x})\right)$ for each predicate $P / n$, open in all definitions of $T ; C_{P}$ is a conjunction of literals and will be called the domain of $P / n$. An example is the FOL axiom $\forall x, y(\operatorname{In}(x, y) \supset E d g e(x, y))$;

- a set of definitions $D_{1}, \ldots, D_{n}$ defining other concepts; the bodies of all rules are conjunctions of literals;

- a set of FOL formulas in the clausal form $\forall\left(A_{1} \wedge \ldots A_{n} \supset B_{1} \vee \ldots \vee B_{m}\right)$, where $A_{i}, B_{j}$ are atoms.

In addition, the definitions of $T$ should satisfy some other conditions. To express these conditions, we need the following concept. The dependency relation $P / n \leq$ $Q / m$ of $T$ is the least transitive relation between predicate symbols containing all pairs $(P / n, Q / m)$ such that $Q / m$ appears in the head and $P / n$ in the body of some rule of some definition $D \in T$. We call an ID-Logic theory $T$ stratified if each predicate is defined in at most one definition of $T$ and any two predicates $P / n$ and $Q / m$ are defined in the same definition of $T$ whenever $P / n \leq Q / m$ and $Q / m \leq P / n$. Given this concept, $T$ should also satisfy the following conditions:

- $T$ is a stratified ID-Logic theory;

- each definition $D \in T$ is total.

A theory $T$ satisfying the above conditions can be easily transformed into a stable logic program $P_{T}$. We describe this transformation in two steps:

- The first step transforms the ID-Logic theory $T$ in an ID-Logic theory $T^{\prime}=$ $T_{a} \cup\left\{D_{T}\right\}$, where $T_{a}$ consists of all FOL axioms of $T$ and $D_{T}=\bigcup_{D \in T} D$, with $\operatorname{Def}\left(D_{T}\right)=\bigcup_{D \in T} \operatorname{Def}(D)$. So, all definitions are merged. Notice that this theory is formally an abductive logic program under the well-founded semantics (where the abducible predicates correspond to $\operatorname{Open}\left(D_{T}\right)$ ).

- In the second step we replace $\neg$ by not, and $\wedge$ by "," in the definition $D_{T}$. Also, we switch case of constant, functor and variable symbols. Using the method of Satoh and Iwayama [15] to transform an abductive logic program into a stable logic program, we then interpret $D_{T}$ as a set of ASP rules, and add to this set the rules

$$
\begin{aligned}
& P(\bar{X}) \leftarrow C_{P}(\bar{X}), \text { not } P^{*}(\bar{X}), \\
& P^{*}(\bar{X}) \leftarrow C_{P}(\bar{X}), \text { not } P(\bar{X})
\end{aligned}
$$

for each predicate $P / n \in \operatorname{Open}\left(D_{T}\right)$. Finally, for each clause $\forall\left(A_{1} \wedge \ldots A_{n} \supset\right.$ $\left.B_{1} \vee \ldots \vee B_{m}\right) \in T_{a}$, we add one rule to the set:

$$
f \leftarrow A, \ldots, A_{n}, \text { not } B_{1}, \ldots, \operatorname{not} B_{m}, \text { not } f .
$$

Theorem 1. An Herbrand interpretation $M$ is a model of $T$ if and only if there is a stable model $M^{\prime}$ of $P_{T}$ such that $M=\left.M^{\prime}\right|_{\tau}$. 
Proof. (sketch) The theory $T$ satisfies the conditions of the modularity theorem of [5]. As a consequence, the ID-Logic theories $T$ an $T^{\prime}$ are equivalent and the definition $D_{T}$ is total. The ID-Logic theory $T^{\prime}$ is formally an abductive logic program under the well-founded semantics. Moreover, because of the totality of $D_{T}$, all well-founded models are 2-valued, and hence the well-founded and stable semantics coincide. The correctness of the last step of the transformation (i.e. from an abductive logic program under the stable semantics to a stable logic program) was proven in [15].

Example 2. The ID-Logic theory of section 1 satisfies the conditions specified in this section. The only non-trivial condition is the totality of the definition of Reached. This definition is a monotone definition (no negative literals with defined predicates in the body of rules) and such definitions are total [5]. The only difference between the translation of this theory and the ASP theory from Fig. 1, is that our translation uses the constraint " $f \leftarrow \operatorname{Vertex}(x)$, not Reached $(x)$, not $f$ ", while the original ASP theory uses " $f \leftarrow$ not reached $(X)$, not $f$ " instead.

\subsection{Extending the transformation}

This section presents an extension of the transformation to a broader class of ID-Logic theories, by providing three separately applicable transformations to theories from the class considered in Section 3.1:

- a transformation from non-stratifiable to stratifiable ID-Logic theories;

- a transformation from arbitrary FOL axioms to clausal axioms;

- a transformation from rules with FOL formulas in the body to conjunctive rules.

Non-stratifiable theories The previous section imposed that no predicate should be defined in more than one definition, and if predicates $P / n$ and $Q / m$ depend on each other, then they are defined in the same definition. If these conditions are not satisfied, then merging together all definitions into one is not equivalence preserving. Example 1 already illustrated this for a non-stratified theory (the theories $[\{P \leftarrow Q\},\{Q \leftarrow P\}]$ and $[\{P \leftarrow Q, Q \leftarrow P\}]$ are not equivalent); the next example shows the problem with multiple definitions for the same concept.

Example 3. Consider the ID-Logic theory

$$
T=\left[\begin{array}{l}
\{\forall x(\operatorname{Person}(x) \leftarrow \operatorname{Man}(x) \vee \operatorname{Woman}(x))\}, \\
\{\forall x(\operatorname{Person}(x) \leftarrow \operatorname{Child}(x) \vee \operatorname{Adult}(x))\}
\end{array}\right]
$$

This theory contains two definitions for the predicate Person. These definitions constrain each other; for example, $T$ logically entails the formula $\forall x(M a n(x) \vee$ $W \operatorname{oman}(x) \equiv \operatorname{Child}(x) \vee \operatorname{Adult}(x))$. If the definitions are merged, this formula is not longer entailed. 
The problems shown are easy to avoid by renaming the defined predicates before merging. We create a new ID-Logic theory $T^{(1)}$ consisting of the following parts:

- for each definition $D \in T$, a definition $D^{\prime}$ obtained from $D$ by replacing any occurrence of $P \in \operatorname{Def}(D)$ by $P^{D}$, where $P^{D}$ is a new predicate;

- all assertions of $T$;

- formulas $\forall \bar{x}\left(P(\bar{x}) \equiv P^{D}(\bar{x})\right)$ for each definition $D$ and predicate $P$ defined in $D$.

Theorem 2. The theory $T^{(1)}$ is a stratified theory. It holds that $T \equiv_{\tau} T^{(1)}$ (where $\tau$ is $T$ 's vocabulary). Moreover if each definition of $T$ is total, then each definition of $T^{(1)}$ is total.

Example 4. The theory $T_{1}$ from example 1 is transformed by this first step into

$$
T_{1}^{(1)}=\left[\begin{array}{ll}
\left\{P^{D_{1}} \leftarrow Q\right\} & P^{D_{1}} \equiv P \\
\left\{Q^{D_{2}} \leftarrow P\right\} & Q^{D_{2}} \equiv Q
\end{array}\right]
$$

Transforming FOL formulas in clausal form The standard transformation of FOL formulas into clausal form cannot be used in this context. The reason is that we assume the Unique Names Axioms while the transformation to CNF introduces skolem function symbols to which the Unique Names Axioms do not apply. Instead, the following variant transformation can be used. Each FOL formula can be brought in the form:

$$
\forall\left(F_{1} \vee \ldots \vee F_{n} \vee G_{1} \vee \ldots \vee G_{m}\right)
$$

where each $F_{i}$ is a literal and each $G_{i}$ is an existentially quantified formula $\exists x H_{i}$.

Let $\bar{x}_{i}$ be all free variables of $G_{i}$. We introduce for each $G_{j}, 1 \leq j \leq m$ a new predicate $P_{i} / n_{i}$ where $n_{i}$ is the number of variables in $\bar{x}_{i}$, and translate the above FOL formula in:

$$
\forall\left(F_{1} \vee \ldots \vee F_{n} \vee P_{1}\left(\bar{x}_{1}\right) \vee \ldots \vee P_{m}\left(\bar{x}_{n}\right)\right)
$$

combined with definitions

$$
\left\{\forall \bar{x}_{i}\left(P_{i}\left(\bar{x}_{i}\right) \leftarrow G_{i}\right)\right\}
$$

Let $T$ be an abritrary ID-Logic theory, and $T^{(2)}$ be the ID-Logic theory obtained by applying the above transformation.

Theorem 3. It holds that $T \equiv_{\tau} T^{(2)}$ where $\tau$ is the vocabulary of $T$ (without the new symbols $\left.P_{i} / n_{i}\right)$. 
Creating conjunctive bodies We now discuss how to transform definitional rules with FOL bodies to rules with conjunctions of literals in the body. The transformation is basically the one proposed by Lloyd and Topor [10], with the exception of the rule for removing universal quantifiers in bodies. Rules of the form

$$
\forall(H \leftarrow \boldsymbol{F} \wedge(\forall x V(x)) \wedge \boldsymbol{G})
$$

are transformed by Lloyd and Topor into a pair of rules

$$
\begin{aligned}
& \forall\left(H \leftarrow \boldsymbol{F} \wedge \neg P^{\prime}\left(y_{1}, \ldots, y_{m}\right) \wedge \boldsymbol{G}\right) \\
& \forall\left(P^{\prime}\left(y_{1}, \ldots, y_{m}\right) \leftarrow \neg V(x)\right)
\end{aligned}
$$

where $y_{1}, \ldots, y_{m}$ are the free variables of $\forall x V(x)$ and $P^{\prime}$ is a new predicate. However, if a predicate depending on $H$ occurs in $V(x)$, this transformation is not equivalence preserving in general. Instead, we replace this rule with

$$
\forall\left(H \leftarrow \boldsymbol{F} \wedge V\left(C_{1}\right) \wedge V\left(C_{2}\right) \wedge \ldots \wedge \boldsymbol{G}\right) .
$$

where $C_{1}, C_{2}, \ldots$ are all terms in the Herbrand universe. Of course, this transformation rule may produce infinitary rules in case the Herbrand Universe is infinite.

The set of all rewrite rules are presented in Figure 2, where $\boldsymbol{F}$ and $\boldsymbol{G}$ denote arbitrary FOL formulae.

By applying the above rewrite rules on an arbitrary ID-Logic-definition $D$ until none is applicable anymore, we obtain a definition $D^{\prime}$ such that all bodies are conjunctions of literals. The following theorem holds.

Theorem 4. The definitions $D$ and $D^{\prime}$ are logically equivalent. Moreover, if $D$ is total then so is $D^{\prime}$.

At this point, we can transform any ID-Logic-theory $T$ into an equivalent IDLogic-theory $T^{(3)}$ which is stratified and such that all bodies of definitional rules are conjunctions of literals. More precisely, it holds that $T \equiv_{\tau} T^{(3)}$. Moreover, if all definitions of $T$ are total, then all definitions of $T^{(3)}$ are total. Consequently, the conditions of theorem 1 hold and $T^{(3)}$ can be transformed into an equivalent stable logic program $P_{T^{(3)}}$. We find that $T \equiv_{\tau} P_{T^{(3)}}$.

\section{A model generator for ID-Logic}

The above transformation, apart from showing the commonalities between methodology of ASP and of ID-Logic, also provides us with an effective means to compute models for a subclass of ID-Logic theories with total definitions: we first translate them, and then apply a stable model generator, such as lparse/Smodels, to the translation.

Since the general tranformation may produce infinitary rules, and/or rules which have to be grounded w.r.t. $\mathcal{H U}(\tau)$, we further restrict the class of ID-Logic theories. 


\begin{tabular}{ll} 
replace & by \\
\hline \hline$\forall \bar{x}\left(H \leftarrow \boldsymbol{F} \wedge\left(\exists y_{1} \ldots y_{m} V\right) \wedge \boldsymbol{G}\right)$ & $\forall \bar{x}, y_{1}, \ldots y_{m}:(H \leftarrow \boldsymbol{F} \wedge V \wedge \boldsymbol{G})$ \\
\hline$\forall \bar{x}\left(H \leftarrow \boldsymbol{F} \wedge \neg\left(\exists y_{1} \ldots y_{m} V\right) \wedge \boldsymbol{G}\right)$ & $\forall \bar{x}\left(H \leftarrow \boldsymbol{F} \wedge \forall y_{1} \ldots y_{m} \neg V \wedge \boldsymbol{G}\right)$ \\
\hline$\forall \bar{x}\left(H \leftarrow \boldsymbol{F} \wedge \neg\left(\forall y_{1} \ldots y_{m} V\right) \wedge \boldsymbol{G}\right)$ & $\forall \bar{x}, y_{1}, \ldots y_{m}(H \leftarrow \boldsymbol{F} \wedge \neg V \wedge \boldsymbol{G})$ \\
\hline$\forall \bar{x}(H \leftarrow \boldsymbol{F} \wedge(V \equiv W) \wedge \boldsymbol{G})$ & $\forall \bar{x}(H \leftarrow \boldsymbol{F} \wedge((V \wedge W) \vee(\neg V \wedge \neg W)) \wedge \boldsymbol{G})$ \\
\hline$\forall \bar{x}(H \leftarrow \boldsymbol{F} \wedge(V \subset W) \wedge \boldsymbol{G})$ & $\forall \bar{x}(H \leftarrow \boldsymbol{F} \wedge V \wedge \boldsymbol{G})$ \\
\hline$\forall \bar{x}(H \leftarrow \boldsymbol{F} \wedge \neg(V \subset W) \wedge \boldsymbol{G})$ & $\forall \bar{x}(H \leftarrow \boldsymbol{F} \wedge \neg W \wedge \boldsymbol{G})$ \\
\hline$\forall \bar{x}(H \leftarrow \boldsymbol{F} \wedge(V \vee W) \wedge \boldsymbol{G})$ & $\forall \bar{x}(H \leftarrow \boldsymbol{F} \wedge V \wedge \boldsymbol{G})$ \\
\hline$\forall \bar{x}(H \leftarrow \boldsymbol{F} \wedge \neg(V \vee W) \wedge \boldsymbol{G})$ & $\forall \bar{x}(H \leftarrow \boldsymbol{F} \wedge W \wedge \boldsymbol{G})$ \\
\hline$\forall \bar{x}(H \leftarrow \boldsymbol{F} \wedge \neg(V \wedge W) \wedge \boldsymbol{G})$ & $\forall \bar{x}(H \leftarrow \boldsymbol{F} \wedge \neg V \wedge \boldsymbol{G})$ \\
\hline$\forall \bar{x}(H \leftarrow \boldsymbol{F} \wedge \neg \neg V \wedge \boldsymbol{G})$ & $\forall \bar{x}(H \leftarrow \boldsymbol{F} \wedge \neg W \wedge \boldsymbol{G})$ \\
\hline \hline & $\forall \bar{x}(H \leftarrow \boldsymbol{F} \wedge V \wedge \boldsymbol{G})$ \\
\hline
\end{tabular}

Fig. 2. Lloyd-Topor transformations for obtaining conjunctive bodies

Definition 1 (Restricted definitions). We define that a definition $D$ is restricted in an ID-Logic theory $T$, by the following inductive rules:

- $D$ is a definition by exhaustive enumeration (of ground facts), or

- D satisfies the following conditions:

- all bodies are conjunctions of literals, and each predicate of the body is defined in a restricted definition in $T$,

- each variable occuring in the head of a rule also occurs in a positive literal of the body of this rule, and

- there is no other definition in $T$ defining predicates defined in $D$

We denote the predicates of an ID-Logic theory $T$ defined in a restricted definition with Restricted $(T)$. If the definitions of restricted predicates are finite, the extension of each restricted predicate is finite and can be computed easily. Consequently, each conjunction $R$ of atoms from Restricted $(T)$ has a finite and computable extension.

To avoid the infinitary rules which are produced in the presence of recursion over $\forall$, we restrict the use of $\forall$ to formulas of the form " $\forall \bar{x}(R(\bar{x}) \supset F(\bar{x}))$ ", where $R$ is a conjunction of restricted atoms, and all free variables of $F(\bar{x})$ occur in $R(\bar{x})$. A rule " $\forall \bar{y}(H \leftarrow \boldsymbol{F} \wedge \forall \bar{x}(R(\bar{x}) \supset F(\bar{x})) \wedge \boldsymbol{G})$ " can be transformed into $" \forall \bar{y}\left(H \leftarrow \boldsymbol{F} \wedge F\left(\overline{C_{1}}\right) \wedge \ldots \wedge F\left(\overline{C_{n}}\right) \wedge \boldsymbol{G}\right)$, where $C_{1}, \ldots, C_{n}$ are all elements of the 
extension of $R$. (Of course, we only do so if $F$ contains a predicate depending on $H$, otherwise we can just use Lloyd-Topor's transformation.)

Definition 2 (Strongly range-restricted ID-Logic theory). An ID-Logic theory $T$ is strongly range-restricted if the following conditions hold (below, $R(\bar{x})$ denotes a conjunction of restricted atoms):

- any definition in it is total;

- all quantifiers in assertions and bodies of rules are restricted to formulas of the form $\forall \bar{x}(R(\bar{x}) \supset F(\bar{x}))$ and $\exists \bar{x}(R(\bar{x}) \wedge F(\bar{x}))$;

- for each open predicate $P$ there is one domain declaration $\forall \bar{x}(P(\bar{x}) \supset R(\bar{x}))$;

- each definitional rule is of the form $\forall \bar{x}(P(\bar{x}) \leftarrow R(\bar{x}) \wedge F)$;

Theorem 5. A strongly range-restricted ID-Logic theory is transformed by our transformation to a strongly range-restricted [16] general logic program.

Note that lparse/Smodels requires strongly range-restricted programs. Therefore, by requiring strongly range-restrictedness of our ID-Logic theories, we can calculate ID-Logic models using lparse/Smodels.

We have devised an implementation of our transformation, which, when applied on theories from this class, and combined with lparse/Smodels, computes ID-Logic models.

\section{Conclusions}

We presented a general tranformation from ID-Logic theories to stable logic programs. This transformation illustrates the fundamental distinction between definitional and assertional knowledge and shows how these can be encoded in Stable Logic Programming. We believe our transformation truthfully corresponds to the way many ASP programs in many applications are developed. This way, the transformation sheds light on the methodologies of ID-Logic and ASP.

Also, the transformation has enabled us to create an ID-Logic model generator, by applying our transformation on an ID-Logic theory, and using an existing ASP model generator to find the models of the resulting program.

\section{References}

1. R. J. Brachman and H.J. Levesque. Competence in Knowledge Representation. In Proc. of the National Conference on Artificial Intelligence, pages 189-192, 1982.

2. M. Denecker. Extending classical logic with inductive definitions. In J. Lloyd et al., editor, First International Conference on Computational Logic (CL2000), volume 1861 of Lecture Notes in Artificial Intelligence, Springer, pages 703-717, 2000.

3. M. Denecker, V.W. Marek and M. Truszczyński. Approximating operators, stable operators, well-founded fixpoints and applications in non-monotonic reasoning. In Logic-based Artificial Intelligence, (J. Minker ed.), Kluwer Academic Publishers, pages $127-144,2000$. 
4. M. Denecker. What's in a model? Epistemological analysis of Logic Programming. In Proceedings of Ninth International Conference on Principles of Knowledge Representation and Reasoning, Delta Whistler Resort, Canada, 2004.

5. M. Denecker and E. Ternovska. A logic of non-monotone inductive definitions and its modularity properties. In Logic Programming and Nonmonotonic Reasoning: 7th International Conference (V. Lifschitz and I. Niemelä, eds.), vol 2923, Lecture Notes in Computer Science, pages 47-60, 2004

6. M. Denecker and E. Ternovska. Inductive Situation Calculus. In Proceedings of Ninth International Conference on Principles of Knowledge Representation and Reasoning, Delta Whistler Resort, Canada, 2004.

7. M. Gelfond. Representing knowledge in A-Prolog. In A. Kakas and F. Sadri, editors, Computational Logic: Logic Programming and Beyond; Essays in honour of Robert A. Kowalski, Part II, number 2407 in Lecture Notes in Computer Science, pages 413-451. Springer Verlag, 2002.

8. M. Gelfond and V. Lifschitz. Logic Programs with Classical Negation. In D.H.D. Warren and P. Szeredi, editors, Proc. of the 7th International Conference on Logic Programming 90, page 579. MIT Press, 1990.

9. M. Gelfond and V. Lifschitz. Classical negation in logic programs and disjunctive databases. New Generation Computing, pages 365-387, 1991.

10. J. W. Lloyd and R. W. Topor. Making Prolog more Expressive. Journal of Logic Programming, 3:225-240, 1984.

11. V.W. Marek and M. Truszczyński. Stable models and an alternative logic programming paradigm. In K.R. Apt, V. Marek, M. Truszczyński, and D.S. Warren, editors, The Logic Programming Paradigm: a 25 Years Perspective, pages 375-398. Springer-Verlag, 1999.

12. I. Niemelä. Logic programs with stable model semantics as a constraint programming paradigm. Annals of Mathematics and Artificial Intelligence, 25(3,4):241-273, 1999.

13. N. Pelov, E. De Mot, and M. Denecker. Logic programming approaches for representing and solving constraint satisfaction problems: a comparison. Proceedings of the 7th International Conference on Logic for Programming and Automated Reasoning, (M. Parigot and A. Voronkov, eds.), vol 1955, Lecture Notes in Artificial Intelligence, pages $225-239,2000$

14. N. Pelov, E. De Mot, and M. Bruynooghe. A comparison of logic programming approaches for representation and solving of constraint satisfaction problems. Proceedings of the 8th International Workshop on Nonmonotonic Reasoning, (C. Baral and M. Truszczyńsky, eds.), pages 1-10, 2000.

15. K. Satoh and N. Iwayama. Computing Abduction by Using the TMS. In Proceedings of ICLP'91, pages 505-518, 1991.

16. T. Syrjänen. Implementation of Local Grounding for Logic Programs with Stable Model Semantics. Helsinki University of Technology, Technical Report, 1998

17. Allen Van Gelder, Kenneth A. Ross, John S. Schlipf. The Well-Founded Semantics for General Logic Programs. Journal of the ACM 38(3):620-650, 1991.

18. W. Woods. What's in a Link: Foundations for Semantic Networks. In D. Bobrow and A. Collins, editors, Representation and understanding: Studies in cognitive science. Academic Press, New York, 1975. Also in Brachman and Levesque, Readings in Knowledge Representation, Morgan Kaufman, 1985. 